\title{
Cloud-Enabled Wireless Body Area Network for Pervasive Health Care
}

\author{
Jarubula Priyanka1)
}

\begin{abstract}
In this project, we tend to primarily finish the support of mobile cloud computing, wireless body space networks may be considerably increased for enormous preparation of pervasive aid applications. However, many technical problems and challenges area unit related to the mixing of WBANs and MCC. During this article, we tend to study a cloud enabled WBAN design and its applications in pervasive aid systems. We tend to highlight the methodologies for sending sign knowledge to the cloud by victimization energy efficient routing, cloud resource allocation, linguistics interactions and knowledge security mechanisms. Pervasive aid applications utilizing body detector networks generate a huge quantity of knowledge that require to be managed and hold on for process and future usage. Cloud computing may be a new trend for economical managing and process of detector knowledge on-line. This paper presents a platform supported cloud computing for management of mobile and wearable aid sensors, demonstrating this fashion the cloud paradigm applied on pervasive aid.
\end{abstract}

Keywords : mobile, cloud computing, WBAN, MCC, security, wearable sensors.

\section{Introduction}

The introduction of the pervasive healthcare paradigm has enabled the notice towards the freelance living of old folks and therefore the want for constant medical direction of chronic patients or habitants at remote, isolated or undeserved locations. During this context, advanced electronic healthcare services area unit needed to be created obtainable through a network time, anywhere and to anyone[1]. A medical helpful setting on the opposite hand issues the employment of pervasive and Omni present technologies for delivering the higher than services[2-5]. Wireless technologies change the important time transmission of knowledge a few patient's condition to caregivers. Varied transportable devices area unit obtainable that may notice bound medical conditions- pulse rate, pressure, breadth alcohol level, so on-form a user's bit[6]. But this development and readying of health data management through mobile devices introduces many challenges: knowledge storage and management(e.g., physical storage problems,

Received(July 1, 2016), Review Result(1st: July 22, 2016, 2nd: August 19, 2016), Accepted(March 10, 2015)

${ }^{1}$ (Corresponding Author) Department of Computer Science and Engineering, KL University email: jarubulapriyanka001@gmail.com 
availableness and maintenance), ability and availableness of heterogeneous resources, security and privacy(e.g., permission management, knowledge obscurity, etc), unified and omnipresent access area unit a couple of to say one potential answer for addressing all same problems is that the introduction of cloud computing conception in electronic health care systems[7-9]. Cloud computing provides the power to access shared resources and customary infrastructure in an exceedingly omnipresent and clear manner, providing services on-demand, over the network, and performing arts operations that meet ever changing desires.

Due to many recent technological advances and new ideas, like wireless body space networks(WBANs) and low power wireless communications, pervasive health observation and management services are getting more and more well liked. However economical management of the big range of monitored knowledge collected from varied WBANs is a very important issue for his or her large scale adoption in pervasive healthcare services. Since WBANs have restricted memory, energy, computation, capabilities, they need a strong and climbable superior computing and big storage infrastructure for real time operation and knowledge storage. Additionally, as far online and offline knowledge analysis. Mobile cloud computing (MCC) is step by step changing into a promising technology, that provides a versatile stack of huge computing, storage, and software system services in an exceedingly climbable and virtualized manner at low value the combination of WBANs and MCC to facilitate the event of efficient, scalable, and data-driven pervasive health care systems, that should be ready to understand long health observation and knowledge analysis of patients in several environments.

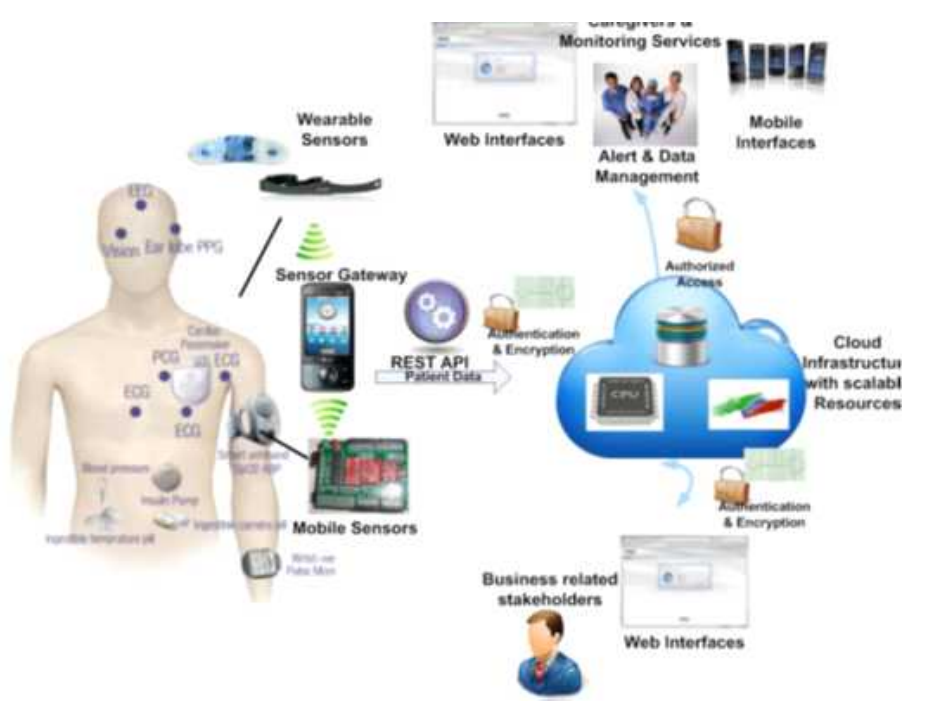

[Fig. 1] Wireless Network 


\section{Proposed system}

\subsection{MCC benefits}

MCC inherits several advantages of cloud computing like dynamic provisioning, quantifiability, and simple integration, yet of a mobile network, like seamless quality. We will develop and develop varied mobile applications for pervasive health care, which might access larger and quicker information storage devices and process power from the cloud. What is more, MCC will improve the responsibility and security of mobile applications, wherever the info and computation will be protected within the medical cloud.

Many mobile applications as well as mobile commerce, mobile learning, and mobile diversion are developed for various MCC environments. As associate example, a example of a mobile healthcare info management system supported cloud computing associated a mobile terminal running an mechanical man software package is being enforced. This example platform is developing services that utilizes the Amazon S3 cloud storage service to manage patient health records and medical pictures.

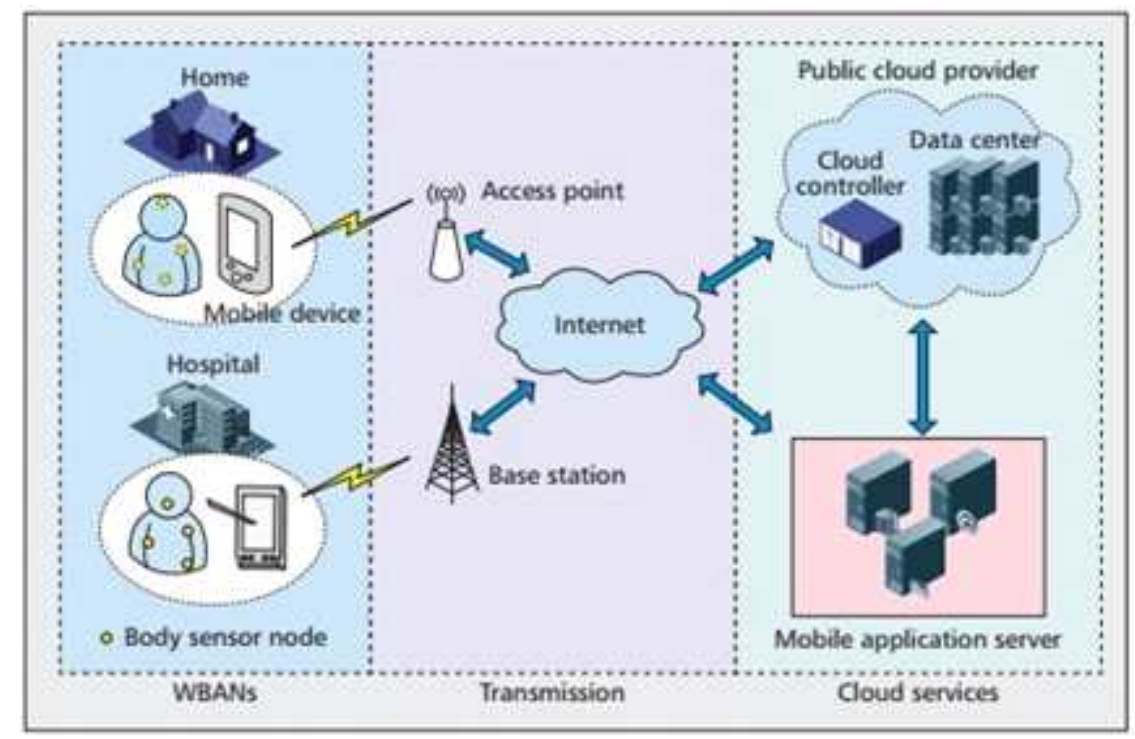

[Fig. 2] Storage Services

The evolving concepts of pervasive computing, ubiquitous computing and close intelligence area unit more and more influencing health care and drugs. Summarizing published analysis, 
this literature review provides an summary of recent developments and implementations of pervasive computing systems in health care. It also highlights some of the experiences rumored in preparation processes. First, systems were included, when they were omnipresent within the sense of being not absolute to one dedicated location, such as a computer at a geographic point. For example, systems of telemedicine via video conferencing at dedicated places were not considered (e.g., stationary desktop computers). Instead, systems were included that featured:

- Existing versus pervasive health care,

- Acute versus continuous,

- Hospitalization versus home and out-patient care,

- Reactive versus pro-active and preventive care,

- IT versus assistive technology,

- centralized versus pervasive,

- $\quad$ Sampling versus monitoring,

- $\quad$ Doctor-centric versus patient-centric.

Having in mind the mentioned development of our societies on the one hand and health-enabling technologies for pervasive health care on the other hand, one may raise, whether pervasive health care is a difficult new field of its own, or if it should higher be regarded as a replacement subfield of medical scientific discipline.

\subsection{Existing system}

The mobile devices serve as gateways for WBANs, and access the Internet via wireless local area network or cellular networks to coordinate with application servers or regionally build choices on the offloading strategy. The mobile devices will then offload the tending tasks to the cloud consequently. Once the requests from patients or mobile application servers have been received, the cloud controllers will schedule the tending tasks on virtual machines (VM), which area unit rented by application service suppliers, and return the results. In some situations, the application servers can even be deployed within the cloud. This system consists of 4 main components: WBANs, wired/wireless transmission, cloud services, and users.

WBANs collect various important signals such as blood heat or pulse rate data from wearable or implantable sensors. The collected monitored data area unit processed in the cloud 
and so by selection transmitted to the users. The medical video streaming from cameras are transmitted to the adjacent routing instrumentation via wired or wireless transmission, and then to the cloud server via the net.

We propose four analysis directions for QoS improvement WMCC platforms, including the development of routing protocol to support economical information transmission to the clouds cloud resource allocation, semantic interactions, and data security and privacy mechanisms. Reliable routing protocols for WBANs must support multi-hop communication and give low end-to-end delay, low packet drop rate, and low energy consumption. Because patients' conditions modification incessantly and might cause large quality problems, new routing protocols may provide various methodology to solve these problems. After work proactive, reactive, and hybrid routing protocols for WBANs, we focus on temperature, cluster-based, and cross-layer routing solutions. Temperature routing focuses on the effects of tissue heating on the frame and their consequences during multi-hop communication. One of the approaches to cut back tissue heating is to reduce the transmission power and traffic rate. Another approach is to always avoid high-temperature nodes once forwarding information packets.

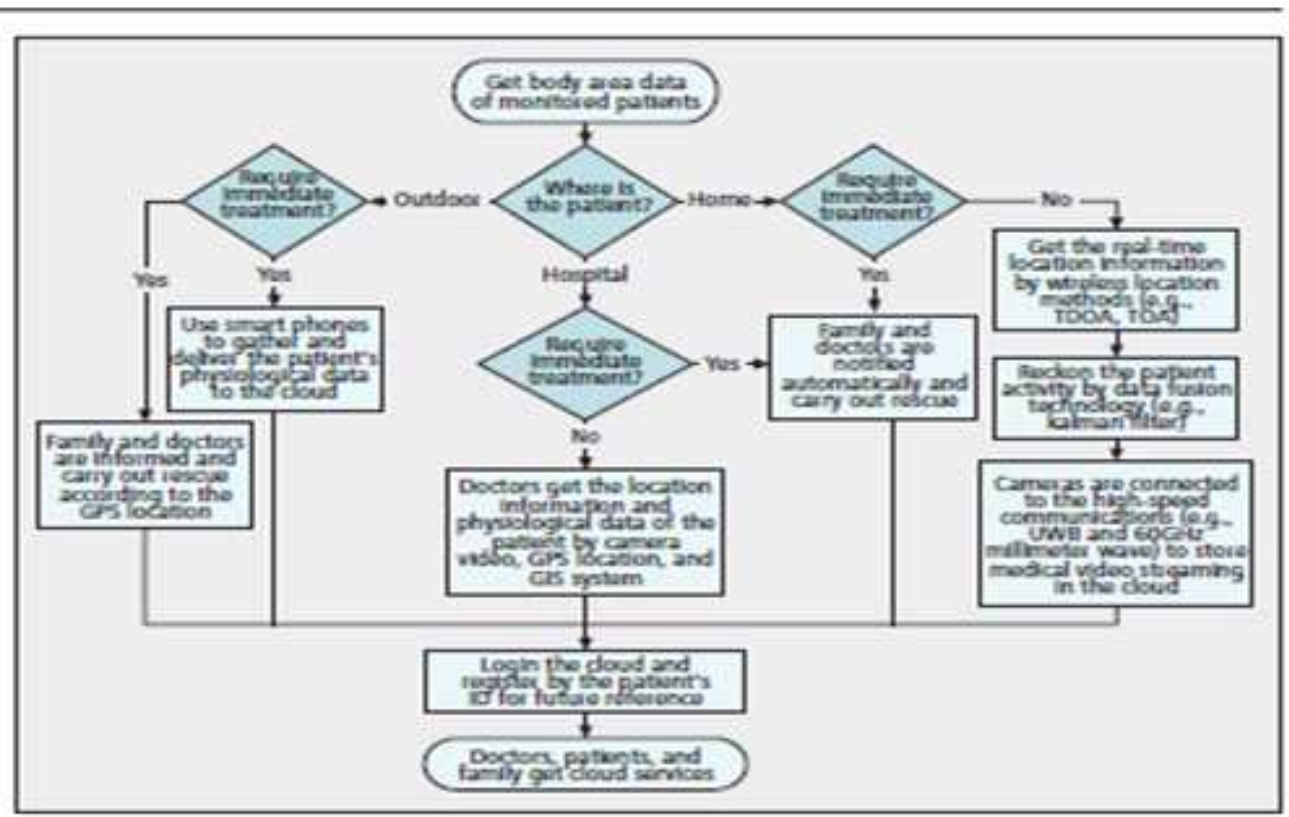

[Fig 3] Logic flowchart of a pervasive healthcare system with MCC capability.

Disadvantages of existing system are: 
Cloud-Enabled Wireless Body Area Network for Pervasive Health Care

- $\quad$ Security

- $\quad$ Storage

- Temperature

- $\quad$ Low energy consumption

Temperature routing focuses on the effects of tissue heating on the flesh and their consequences during multi-chip communication. One of the approaches to cut back tissue heating is to reduce the transmission power and traffic rate. Another approach is to always avoid high-temperature nodes once forwarding knowledge packets.

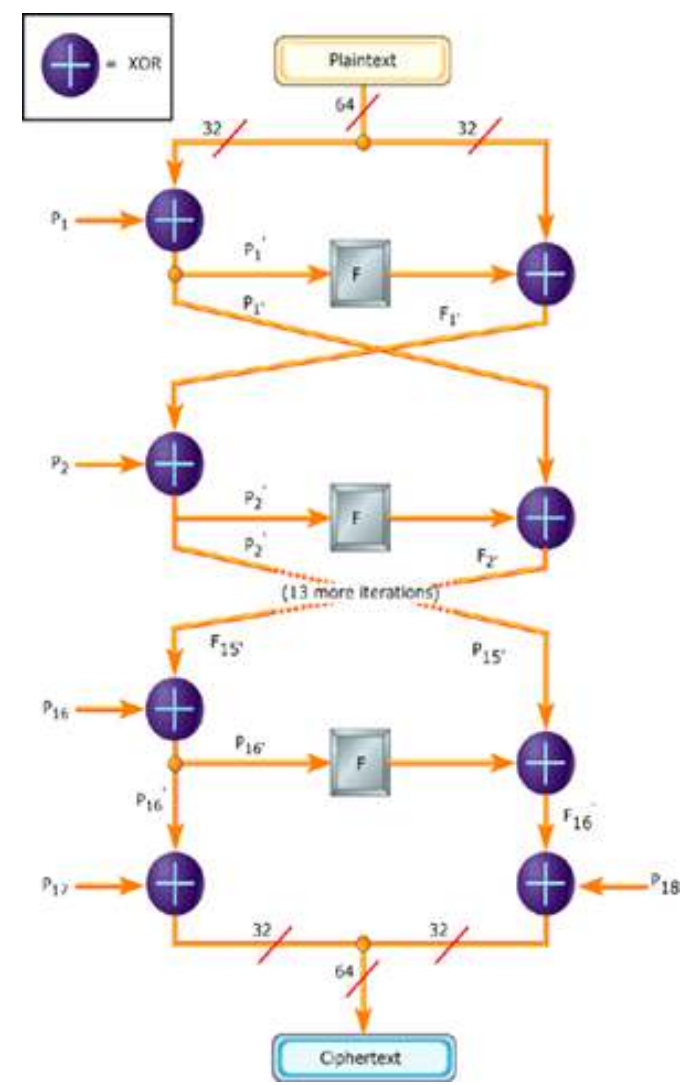

[Fig. 4] Encryption flow of 32-bit processor

In extreme heterogeneous cloud-enabled WBANs, all kinds of resources, such as bandwidth, computing, storage, software, and data resources, are integrated to give data and application service to users. Semantic models will give a set of generic normal protocols for heterogeneous and distributed computing. Semantic models area unit helpful for pervasive health care cloud 
service from the aspects of useful definition, data model, service description enhancement, and so on. The information with the linguistics is straightforward to grasp and method for the pc within the pervasive health care service. The ontology technology is used to implement data linguistics interaction in cloud-enabled WBANs.

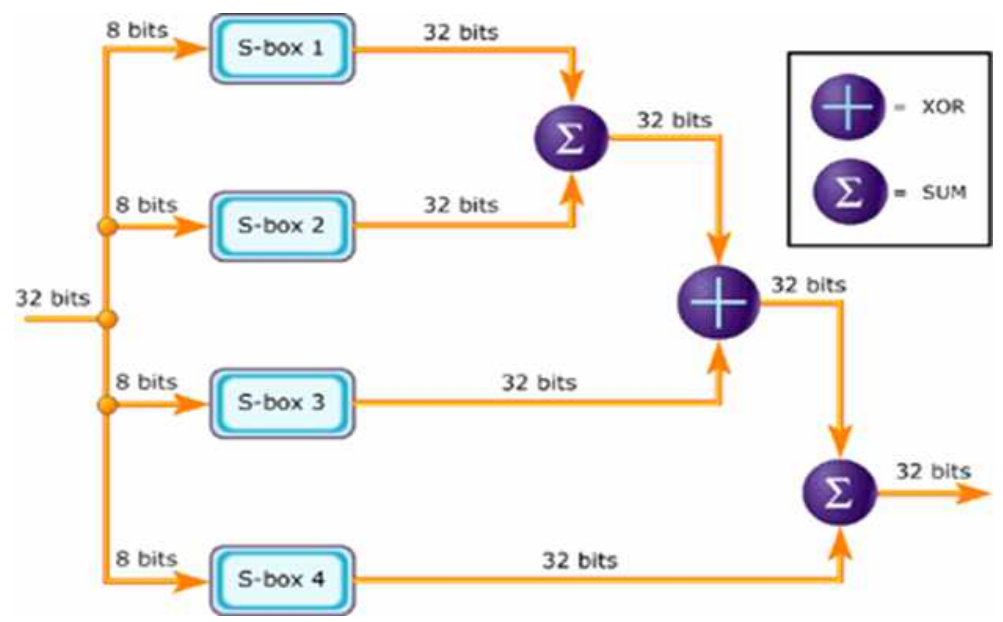

[Fig. 5] 32 bit cipher text flowchart with XOR and SUM nodes

In this description, a 64-bit plaintext message is first divided into thirty two bits. The "left" 32 bits area unit XOR ED with the initial part of a P-array to form a price i will decision $\mathrm{P}^{\prime}$, run through a transformation function referred to as $\mathrm{F}$, then XOR ed with the "right" thirty two bits of the message to turn out a brand new worth $\mathrm{i}$ will decision $\mathrm{F}^{\prime} \cdot \mathrm{F}^{\prime}$ then replaces the "left" [*fr1] of the message and $\mathrm{P}^{\prime}$ replaces the "right" half, and the process is recurrent fifteen additional times with serial members of the P-array. The resulting $\mathrm{P}^{\prime}$ and $\mathrm{F}^{\prime}$ area unit then XOR finish with the last 2 entries in the P-array (entries seventeen and 18), and recombined to produce the 64-bit cipher text.

The P-array and S-array values used by Blowfish are pre computed supported the user's key. In effect, the user's key is transformed into the P-array and S-array; the key itself could also be discarded once the transformation. The P-array and S-array need not be re computed.

\section{Conclusion}

Pervasive healthcare applications generate a large quantity of detector knowledge that require to be managed. Properly for further analysis and process. Cloud computing through its elasticity and facility to access shared resources and common infrastructure in a very present 
and pervasive manner could be a promising answer for economical management of pervasive health care knowledge. The presented system is distinctive as an ardent answer for managing patient-related knowledge on the cloud which utilizes each open hardware and open code resources for developing the hardware and code components of the platform. The seamless integration of WBANs and MCC provides tremendous opportunities for pervasive healthcare systems. In this article, we offer a quick review and outlook of this promising field, and discuss a cloud-enabled WBAN architecture for pervasive health care systems. In particular, we study the practicality and dependability of MCC services. We conjointly counsel some future analysis directions to improve performance and QoS of cloud-enabled WBANs. We believe cloud-enabled WBANs can attract monumental attention and analysis effort within the close to future.

\section{References}

[1] U. Varshney, Pervasive Healthcare, IEEE Computer Magazine, (2003), Vol.36, No.12, pp.138-140.

[2] I. Maglogiannis, C. Doukas, G. Kormentzas, and T. Pliakas, Wavelet- Based Compression With ROI Coding Support for Mobile Access to DICOM Images Over Heterogeneous Radio Networks, IEEE Transactions on Information Technology in Biomedicine, (2009), Vol.13, No.4, pp.458-466.

[3] http://www.gogrid.com, August 25 (2016).

[4] J. Zhou, Z. Cao, X. Dong, N. Xiong, and A. V. Vasilakos, 4S: A secure and privacy-preserving key management scheme for cloud-assisted wireless body area network in m-healthcare social networks, Information Sciences, (2015), Vol.314, pp.255-276.

[5] L. Gatzoulis and I. Iakovidis, Wearable and portable E-health systems, IEEE Eng. Med. Biol. Mag., (2007), Vol.26, No.5, pp.51-56.

[6] Y. Jiang, C. Lin, X. Shen, and M. Shi, Mutual authentication and key exchange protocols for roaming services in wireless mobile networks, IEEE Trans. Wireless Commun., (2006), Vol.5, No.9, pp.2569-25476.

[7] C. C. Y. Poon, Y. Zhang, and S. Bao, A novel biometrics method to secure wireless body area sensor networks for telemedicine and m-health, IEEE Commun. Mag., (2006), Vol.44, No.4, pp.73-81.

[8] Y. Ren, R. W. N. Pazzi, and A. Boukerche, Monitoring patients via a secure and mobile healthcare system, IEEE Wirel. Commun., (2010), Vol.17, No.1, pp.59-65.

[9] Y. Zhou and Y. Fang, Scalable and deterministic key agreement for large scale networks, IEEE Trans. Wireless Commun., (2007), Vol.6, No.12, pp.4366-4373. 